\title{
MRI Signal Intensity Changes of Vertebral Bone Marrow
}

\author{
Divya G. ${ }^{1}$, Ankamma Rao D. ${ }^{2}$ \\ ${ }^{1}$ Department of Radiodiagnosis, NRI Medical College and Hospital, Chinakakani, Andhra Pradesh, India. ${ }^{2}$ Department \\ of Radiodiagnosis, NRI Medical College and Hospital, Chinakakani, Andhra Pradesh, India.
}

\section{ABSTRACT}

\section{BACKGROUND}

The main purpose of the article is to review the normal vertebral marrow and its appearance on MRI, including age-related changes and pathologic appearance based on routine MR sequences like T1, T2 \& STIR. The signal intensity of the bone marrow depends mainly on the fat and water content. Each component has its appearance on MRI sequence that allows their differentiation. The red marrow is highly cellular, so it leads to a low signal intensity on T1-weighted sequences and high signal intensity on short tau inversion recovery (STIR) or fat-saturated T2-weighted sequences. Whereas, the yellow marrow presents an increased signal on T1- weighted sequences and low signal intensity on STIR or fat-saturated T2-weighted sequences. The differential diagnosis of pathological bone marrow includes degenerative changes, neoplasm, infection, and infiltrative marrow disorders. In this study, 30 cases were included, who presented with a chief complaint of neck pain, backache and radiculopathy who underwent MRI for evaluating the underlying pathology. The main objective of this study is to evaluate different MRI findings in these patients.

\section{METHODS}

Patients having neck pain, backache, and radiculopathy who presented in the Department of Radiodiagnosis at NRI Medical College and Hospital, Chinakakani, were included in the present study. On a random basis, 30 cases were included in the study, and all of them underwent routine MRI spine to a particular region according to the symptom.

\section{RESULTS}

Thirty patients were studied with an age range of 22 years to 80 years. In our study, out of 30 patients, 4 had fatty replacement of marrow, 3 had hemangioma, 6 had Modic endplate changes, 3 had metastases, 4 had osteoporosis, 5 had Koch's spine, 5 had myeloproliferative disease. Of the 4 patients who had osteoporosis, two patients had wedge compression fractures.

\section{CONCLUSION}

MRI is the sensitive tool in the imaging of vertebral marrow and imaging modality of choice in studying the bone marrow diseases. Routine sequences like T1, T2 \& STIR sequences are the key for proper diagnosis and management of spinal pathology.

\section{KEY WORDS}

Vertebral Bone Marrow, Signal Intensity, MRI, T1 \& T2 Weighted Imaging
Corresponding Author:

Dr. Divya G.,

Room No. 253,

NRI Girls Hostel,

Chinakakani-522503,

Mangalagiri, Andhra Pradesh, India.

E-mail:drdivyagullipalli@gmail.com

DOI: $10.14260 /$ jemds $/ 2020 / 52$

Financial or Other Competing Interests: None.

How to Cite This Article:

Divya G, Rao AD. MRI signal intensity changes of vertebral bone marrow. J. Evolution Med. Dent. Sci. 2020;9(04):227230, DOI: $10.14260 /$ jemds $/ 2020 / 52$

Submission 01-11-2019,

Peer Review 07-01-2020,

Acceptance 14-01-2020,

Published 27-01-2020. 


\section{BACKGROUND}

Vertebral bone marrow is important in the evaluation of spine MR imaging. The MRI appearance of the bone marrow is determined by the relative amount of protein, water, fat, and cells within and depends on the pulse sequence on which it is being evaluated. The bone marrow components are the trabeculae, the red marrow and the yellow marrow. The architectural support for the bone marrow is the trabeculae, and its main function is to be the mineral depot. The bone density decreases with age. The major determinants of the appearance of marrow are the fat and water content. Red marrow contains hematopoietic cellular elements supporting the stroma and vascular supply and characterized by containing approximately $40 \%$ fat and its hematopoietic function, whereas yellow marrow contains $80 \%$ fat and small fraction of red marrow elements with poor vascular supply. ${ }^{1}$

The routine spine evaluation on MRI typically includes T1weighted, T2-weighted, and STIR sequences. T1-weighted images are best to evaluate the cellular content in bone marrow because of high-fat content interspersed with hematopoietic elements. Yellow marrow has signal intensity comparable with subcutaneous fat, whereas red marrow has intermediate signal intensity lower than subcutaneous fat but higher than disc or muscle. On T2- weighted images as fat and water have similar signal intensity; there is decrease contrast of marrow in this sequence. The STIR sequence is very sensitive to evaluate vertebral marrow for oedema from contusion/fracture or degeneration or cellular marrow changes related to neoplasm.

\section{METHODS}

This is the retrospective study. As the patients are not directly involved in this study, IEC clearance is not required. 30 patients having a chief complaint of neck pain, backache, and radiculopathy presented in the Department of Radiodiagnosis at NRI Medical College and Hospital, Chinakakani, from February 2019 to July 2019 were included in the present study. The inclusion criteria were presented with neck pain, low backache, and radiculopathy and who were ready to undergo MR imaging. The patients who are not willing to participate in the study, claustrophobic patients, and patients having any metallic/electronic implants in the body were excluded.

\section{RESULTS}

Thirty patients were studied with an age range of 22 to 80 years, with a mean age of 47.88 years. In our study, out of 30 patients, 11 were females, and 19 were males (Figure 1). Thirty patients with the chief complaint of neck pain, low backache, and radiculopathy were studied with an age range of 22 to 80 years. In our study, the maximum number of cases are males. A maximum number of cases were observed in the mean age group of 45-50 years. Of all, two-thirds of the cases are presented with neck pain and low backache. Among all cases were diagnosed by MRI, 4(13.3\%) had patchy fatty replacement of marrow which is an age-related change, $3(10 \%)$ had haemangioma includes typical and atypical types, $6(20 \%)$ had Modic endplate changes, $3(10 \%)$ had metastases of focal and diffuse involvement in which one patient had pathological fracture, 4(13.3\%) had osteoporosis in which 2 patients had wedge compression fracture, 5(16.7\%) had Koch's spine with adjacent soft tissue component, 5 (16.7\%) had myeloproliferative disorders (Figure 2). Most of the pathologies were observed in the $5^{\text {th }}-6^{\text {th }}$ decade.
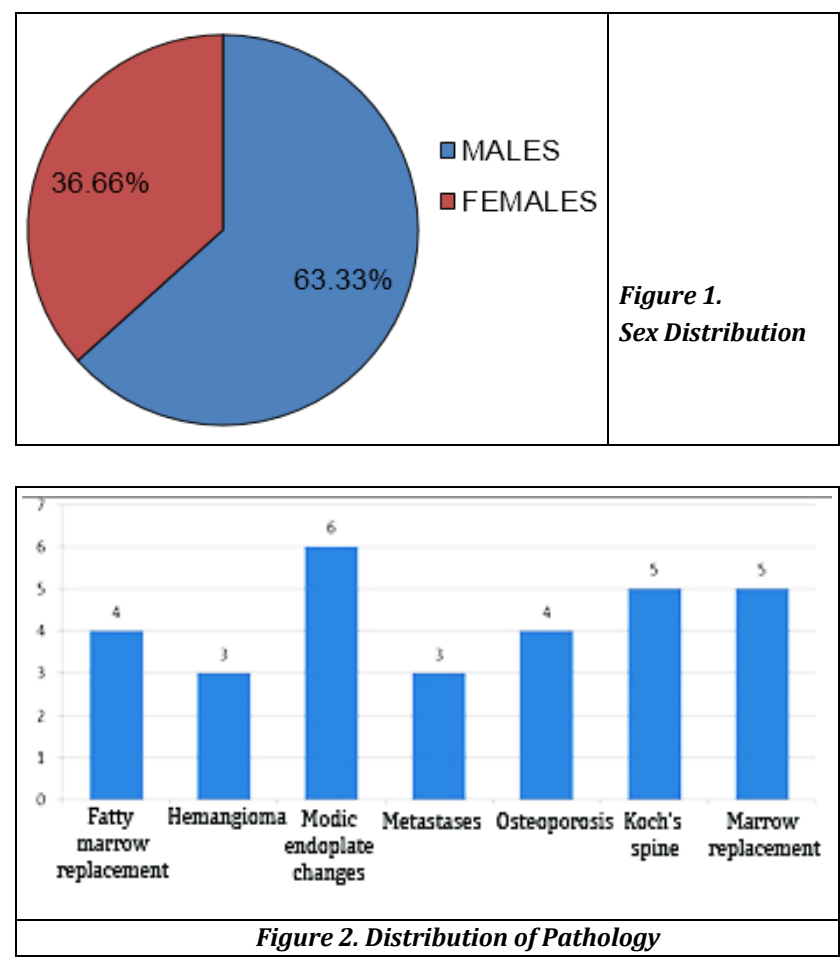

\section{DISCUSSION}

The imaging appearance depends on the composition of fat and water. The fat composition determines the maturation of the bone marrow. The distribution of bone marrow components is modified with age, physiologic stress and the presence of pathology.

As the age of the patient increases, there is fatty conversion of the marrow with low marrow cellularity. It is characterized by a particular distribution pattern within the skeleton: it starts in the peripheral skeleton and progress centrally. At birth, all the marrow is hematopoietic, which is highly cellular or red marrow, which has $40 \%$ macroscopic fat, and with aging, there is conversation to fatty marrow with $80 \%$ macroscopic fat and marrow begins in distal extremities. The conversion completes between 20-25 years when the red marrow retained in the axial skeleton and proximal metaphyses of long bones. The conversion of fat occurs adjacent to the basivertebral plexus between the adolescence and 40 years. After 40 years of age, conversion occurs throughout the remainder in one of the three patterns: (1) Micronodular, (2) Macronodular, and (3) Peripheral, followed by (4) Nodular pattern.2

Spinal haemangiomas are one of the most frequently encountered benign focal marrow abnormalities. They are 
estimated to be present in $27 \%$ of lumbar spine MR imaging examinations, and approximately one-third of haemangiomas are multifocal. ${ }^{3}$ Lesions are most frequently encountered in the vertebral bodies but can extend into the posterior elements. They result due to enlarged vascular spaces and proliferation of fatty stroma- appears hyperintense on T1 \& T2 weighted images and hypointense in fat suppression sequence (STIR) (Figure 3).Vertebral body haemangiomas have also been described as having a "polka-dot" or "corduroy" pattern on CT. ${ }^{4}$ Atypical haemangioma has hypointense signal on T1 and hyperintense signal on T2 \& STIR due to hypocellularity.

Degenerative endplate changes follow an imaging pattern of 3 types 2 : Type 1- T1 hypointense and T2 hyperintense due to oedema in the adjacent marrow. Type 2- T1 and T2 hyperintense, due to fatty marrow proliferation as a result of chronic ischemic change. Type 3- $\mathrm{T} 1$ and $\mathrm{T} 2$ hypointense due to sclerosis of the endplates. The bone marrow pathologies are classified according to common pathophysiological patterns into different groups: replacement disorders, vascular disorders, infiltrative disorders, and depletion disorders ${ }^{2}$. The replacement disorders are due to the infiltration of the bone marrow by cells that do not normally exist, for example, in osteomyelitis, primary bone tumours, lymphomas, or metastasis. The MRI pattern of replacement disorders is similar to infiltrative disorders. Metastases have focal or diffuse involvement of the bone marrow. Pathological fracture can be seen in focal involvement. Osteoporosis is a diffuse marrow replacement entity in which there will decrease bone mass and altered microarchitecture, leading to increased fragility and fracture. The cellular marrow contents are replaced by intramedullary fat. On imaging, there will be diffuse $\mathrm{T} 1 \& \mathrm{~T} 2$ heterogeneous signal intensity due to decreased cellular marrow and increased fat component. Several findings of the osteoporosis range from presence of subchondral lobules of fat, accentuation of vertical and horizontal marrow lines or dotted foci of high signal intensity on T2-weighted fat-suppressed sequences. ${ }^{2}$

\begin{tabular}{|c|c|}
\hline Benign Fracture & Pathological Fracture \\
\hline $\begin{array}{l}\text { Linear bone marrow signal } \\
\text { abnormality at the site of fracture with } \\
\text { normal surrounding marrow, and } \\
\text { Presence of retropulsion of fracture } \\
\text { fragment. }\end{array}$ & 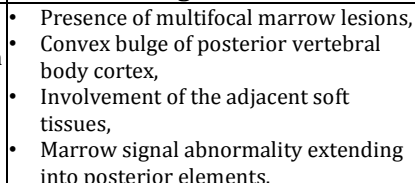 \\
\hline & Fracture (Osteoporotic) and \\
\hline
\end{tabular}

The vascular disorders are due to oedema or ischemia. The bone marrow oedema is the most frequent cause due to an increase in water content, which produces decreased signal intensity on T1- weighted sequences and an increased signal on fat-saturated T2 and STIR sequences. The bone marrow oedema can be found in trauma (bone contusions and stress fractures), migratory osteoporosis, bone marrow oedema syndrome, tumours, sympathetic reflex dystrophy, infections, early osteonecrosis, and articular affections. ${ }^{2}$ Bone marrow ischemia favours fatty marrow over hematopoietic marrow, due to the limited vascular supply of yellow marrow relative to the red marrow. The ischemia can be caused by trauma, steroid treatment, sickle cell disease. In infective spondylodiscitis, infection typically spreads from the endplates to adjacent discs, marrow spaces, and adjacent soft tissues. Infection tends to spare posterior elements most often. ${ }^{1}$ Early findings include endplate erosions and oedema and later intervertebral marrow and disc abnormalities, paraspinal inflammation, and eventually trans-spatial spread into epidural and adjacent soft tissues. In the infiltrative disorders, there is a proliferation of the native cells in the marrow due to benign or malignant conditions. The most common benign process in infiltrative disorders is marrow reconversion or myeloid hyperplasia. In physiological stress, the yellow marrow may get reconverted to red marrow to supply a functional demand to increase the haematopoiesis. The reconversion can be caused by smoking, long-distance running, obesity, middle-age women, anaemia, chronic diseases, or after chemotherapy or radiotherapy (in the nonirradiated bone).1,2 In leukemia, the infiltration of the marrow is typically diffuse, resulting in a diffuse decrease in marrow signal intensity on T1-weighted sequences. In multiple myeloma, the infiltrative pattern on MR is variable, ranging from normal to focal or diffuse.

\section{Classification of Multiple Myeloma2:}

1. Non visualized disease

2. Micronodular ("Variegated" or Salt and Pepper)

3. Multifocal

4. Diffuse marrow infiltration.

On imaging marrow is hypointense on both $\mathrm{T} 1$ \& $\mathrm{T} 2$ weighted images. Although the diffuse disease may mimic the extensive red marrow reconversion, the signal intensity on fat suppressed T2 sequences is generally considerably higher than that of the muscle. ${ }^{3}$ The marrow depletion is due to the loss of normal red marrow that could be regionally or diffusely. It is most commonly caused by radiotherapy, chemotherapy, or aplastic anaemia. In cases of aplastic or hypoplastic marrow, imaging there is diffuse high signal intensity on $\mathrm{T} 1$ and $\mathrm{T} 2$ sequences and low signal intensity on STIR sequences. The effect of radiotherapy is marrow suppression, which depends on the radiation dose, the treatment frequency, and the volume of marrow treated. These bone marrow changes depend directly on the radiation dose.

Acute phase (day 1 to 3 of radiation), the marrow develops oedema, which appears hypointense on $\mathrm{T} 1$ sequences and hyperintense on fat-saturated T2 \& STIR sequences. Upon the gadolinium administration, it presents a transiently increased enhancement on T1- weighted sequences. Later, on 4-10 days, focal areas of haemorrhage can be detected (which are hyperintense on $\mathrm{T} 1$ weighted and hypointense on $\mathrm{T} 2$ weighted/ STIR images). By the third and sixth weeks of the treatment, the pattern of high signal on STIR images decreases by time at this point bone marrow signal on T1 sequences increases, which corresponds to the fatty marrow. After the sixth week, the majority of patients will have a hyperintense T1 signal (i.e., fatty marrow), which can last up to two years. In the dose range of 3-45 Gy used in local irradiation causes rapid bone marrow alteration, which persists up to 2 years. ${ }^{4}$ Regeneration of the marrow is seen with local radiation doses below 30 Gy, while doses above 50 Gy will result in marrow ablation. ${ }^{4}$ 


\section{CONCLUSIONS}

MRI is a sensitive tool in the imaging of vertebral marrow and imaging modality of choice in studying the bone marrow diseases. Routine sequences like T1, T2 \& STIR sequences are the key for the proper diagnosis and management of spinal pathology.

\section{REFERENCES}

[1] Shah LM, Hanrahan CJ. MRI of spinal bone marrow: part 1, techniques and normal age-related appearances. Am J Roentgenol 2011;197(6):1298-308.
[2] Leake RL, Mills MK, Hanrahan CJ, et al. Spinal marrow imaging: clues to disease. Radiol Clin North Am 2019;57(2):359-75.

[3] Barzin M, Maleki I. Incidence of vertebral hemangioma on spinal magnetic resonance imaging in Northern Iran. Pak J Biol Sci 2009;12(6):542-4.

[4] Hanrahan CJ, Shah LM. MRI of spinal bone marrow: part 2, T1-weighted imaging-based differential diagnosis. Am J Roentgenol 2011;197(6):1309-21. 